\title{
SIMPLE MICROMECHANICAL MODEL OF PROTEIN CRYSTALS FOR THEIR MECHANICAL CHARACTERIZATIONS
}

\author{
Gwonchan Yoon, Kilho Eom ${ }^{*}$, and Sungsoo $\mathrm{Na}^{\dagger}$ \\ ${ }^{1}$ Department of Mechanical Engineering, Korea University \\ Seoul 134-701, Republic of Korea
}

\begin{abstract}
Proteins have been known to perform the excellent mechanical functions and exhibit the remarkable mechanical properties such as high fracture toughness in spider silk protein [1]. This indicates that the mechanical characterization of protein molecules and/or crystals is very essential to understand such remarkable mechanical function of protein molecules. In this study, for gaining insight into mechanical behavior of protein crystals, we developed the micromechanical model by using the empirical potential field prescribed to alpha carbon atoms of a protein crystal in a unit cell. We consider the simple protein crystals for their mechanical behavior under tensile loading to be compared with full atomic models
\end{abstract}

\section{Introduction}

Mechanical characterization of proteins have recently enabled the understanding of their mechanical and/or biological functions. Moreover, the studies on mechanical functions of proteins have recently allowed for gaining insight into how to design the novel materials which can perform the excellent mechanical functions based on biomimetics. Specifically, the remarkable nonlinear elastic behavior of biological gels such as actin, collagen, and neurofilaments have been explained by network model of polymer chains [2]. Nevertheless, such model is a phenomenological model available when experimental data exists, and such a model does not provide the relationship between mechanical properties of proteins and their molecular structures such as mutants. As a consequence, the molecular models for mechanical characterization of proteins based on their molecular structures have taken intensively considered in recent decades [3]. Most of molecular models have been based on the molecular dynamics (MD) simulation which requires the a lot of computational cost for large spatial and temporal scales, leading to the computational limitations on studying protein mechanics. Furthermore, MD simulations for mechanical characterization of proteins allows for depicting the microscopic mechanical behavior in the single-molecule level [3]. This indicates that the macroscopic mechanical behavior of proteins have been limited by current MD simulations. In this short paper, we consider the micromechanics model for mechanical behavior of proteins, based on periodic boundary conditions and Virial stress formulations. It is shown that our micromechanical model is able to describe the macroscopic mechanical behavior of beta-sheet. This sheds light on a micromechanics model for describing the mechanical behavior of proteins.

\footnotetext{
*E-mail: kilhoeom@gmail.com

†E-mail: nass@korea.ac.kr
}

This is an Open Access article distributed under the terms of the Creative Commons Attribution-Noncommercial License 3.0, which permits unrestricted use, distribution, and reproduction in any noncommercial medium, provided the original work is properly cited. 


\section{Method}

For mechanical characterization, we consider the representative volument element (RVE)[4] which is applied by periodic boundary condition such as

$$
u_{i}\left(x_{i}+h e_{i}\right)=\varepsilon_{i j}^{0} h e_{j}
$$

where $x_{i}$ is the coordinates of a point in RVE, $h$ is a length-scale of RVE, $e_{i}$ is the Bravis lattice vector, and $\varepsilon_{i j}{ }^{0}$ is a constant strain field applied to the boundary of RVE. The micromechanics theory provides that the macroscopic strain (overall strain) $<\varepsilon_{i j}>$ is equal to a constant strain $\varepsilon_{i j}{ }^{0}$. Once the overall stress $\left\langle\sigma_{i j}>\right.$ is computed from molecular model with boundary condition given by Eq. (1), then the material properties for protein crystal can be decticated as $\left\langle\sigma_{i j}>=C_{i j k j}<\varepsilon_{k l}\right\rangle$, where $C_{i j k j}$ is the 4th order tensor representing the material properties of crystal.

An unitcell consists of several protein molecules. Each $\alpha$-carbon atoms $\left(C_{\alpha}\right)$ are governed by potential field, which is a sum of various energies arising from every covalent bonding length, angle, dihedral angles, nonbonded interaction and hydregen bond energies and so on. Among of these energies we use simply covalent bonding and nonbonded interaction, of which is called Go's potential field [5]. The potential of covalent bonding is represented as

$$
V_{B B}=\sum_{i=1}^{N-1}\left[k_{1}\left(r_{i, i+1}-d_{0}\right)^{2}+k_{2}\left(r_{i, i+1}-d_{0}\right)^{4}\right]
$$

where $r_{i, i+1}=\left|r_{i}-r_{i+1}\right|$ is the distance between residue $i, i+1, d_{0}=3.8 \AA$ is the equilibrium covalent bond length, $k_{1}=n / \AA^{2}, k_{2}=100 \backsim \AA^{4}$ is characteristic force constant. The non bonded interaction is Lennard-Jones potential,

$$
V_{N A T}=\sum_{i<j}^{N A T} 4 \eta\left[\left(\frac{\sigma_{i j}}{r_{i j}}\right)^{12}-\left(\frac{\sigma_{i j}}{r_{i j}}\right)^{6}\right]
$$

The nonbonded energy displays a typical minimum energy $n$ at a distance $\left(\sigma_{i j}=5 \AA\right)$ nearly equal to the sum of van der Waals raddi of the two carbon atoms.

For energy minimization, we perform the conjugate gradient method, 1st order method [6]. The initial guess is proposed an uniaxial strain applied at unitcell. From the Virial theorem, we calculate interatomic stress [7]. When $\alpha$ pair atoms are in volume $v$, the stress is represented as the sum of product of vector connecting the pair force associated with the pair.

$$
v \boldsymbol{\sigma}_{\mathrm{ij}}=\sum_{\alpha} \mathbf{F}_{\mathbf{i}}(\alpha) \mathbf{r}_{\mathbf{j}}(\alpha), \quad i, j=1,2,3
$$

The pressure is defined as

$$
p=-\frac{1}{3}\left(\sigma_{11}+\sigma_{22}+\sigma_{33}\right)
$$




\section{Result and Discussion}

We choose two types of protein, $\alpha$-helix and $\beta$-sheet to validate the simple protein model by comparing the mechanical behavior of $\alpha$-helix with one of $\beta$-sheet. The $\alpha$-helix has coiled structure, of which each turn is weak hydrogen-bonded, while in $\beta$-sheet, hydrogenbond is formed only between strands. To observe this trend, we use one model, $\alpha$ conotoxin PnIB from Conus pennaceus (PDB ID: $1 \mathrm{akg}$ ), which has $\mathrm{P} 2{ }_{1} 2_{1} 2_{1}$ space group (Fig. 1). The volume of an unitcell is $14.6 \AA \times 26.1 \AA \times 29.2 \AA$. The other is MVGGVV peptide derived from Alzheimer's A-beta (2ona) of which space group is $\mathrm{P}_{1}$ and its unitcell volume is $25.89 \AA \times 9.7 \AA \times 15.85$.
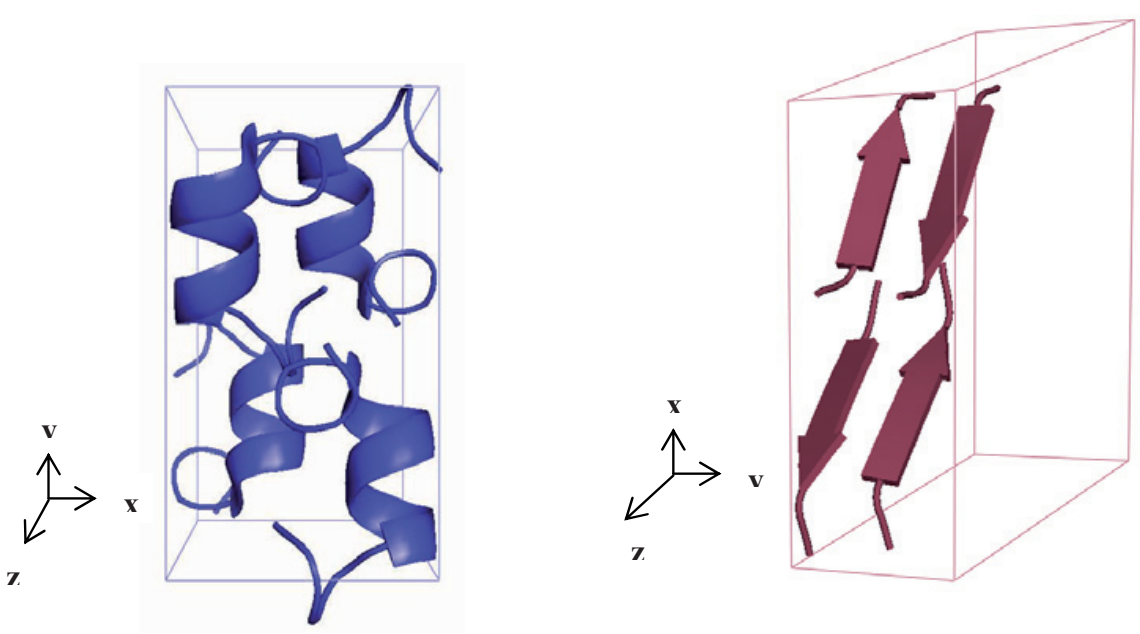

Fig. 1 A cartoon of an unitcell of 1akg (left) and 2ona (right)

Uniaxial strain was applied to each unitcell, respectively, and we obtained the corresonding pressure-strain curve of $\alpha$-helix (Fig. 2.) and $\beta$-sheet (Fig. 3.).

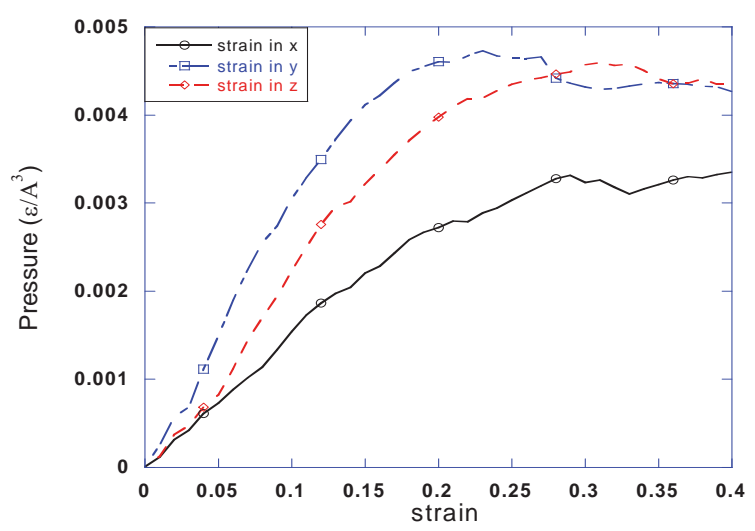

Fig. 2 Pressure-strain curve of $\alpha$-helix 
From the results of tensile test on protein, we can see the characteristic of elastic-plastic material including strain hardening effect. The fracture phenomenon occured when slope of curve has changed. We can check the ultimate stress of $\beta$-sheet (about $0.017 \varepsilon / \AA^{3}$ in $\mathrm{x}$, longitudinal direction) is larger than of $\alpha$-helix (about $0.0045 \varepsilon / \AA^{3}$ in y).

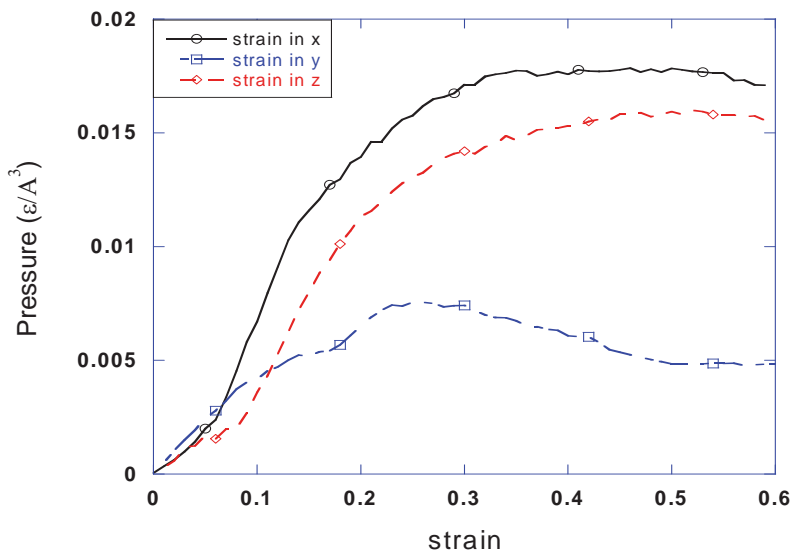

Fig. 3 Pressure-strain curve of $\beta$-sheet.

\section{Conclusion}

We presented the macroscopic mechanical behavior of major protein crystals from the tensile loading conditions. We could observe elastic-plastic property, fracture phenomenon of protein crystals under ultimate tensile stress. Furthermore simple protein crystal model was validated to compare the mechanical properties of $\alpha$-helix and $\beta$-sheet. It is expected that we obtain explicit property of protein such as Young's modulus, shear modulus etc. from further study. Finally, proposed simple model will be extended to compare with experimental data for nonlinear elastic behavior of biological gels.

\section{Acknowledgements}

This work was supported by Basic Science Research Program of the National Research Foundation of Korea funded by the Ministry of Education, Science and Technology under grant No. 2009-0063170 and KRF-2008-314-D00012.

\section{References}

1. NATHAN BECKER, E. O., STEPHANIE MUTZ, JASON P. CLEVELAND, PAUL K. HANSMA, CHERYL Y. HAYASHI, DMITRII E. MAKAROV AND HELEN G. HANSMA* (2003). "Molecular nanosprings in spider capture-silk threads." Nature material 2: 278-283.

2. Cornelis Storm, J. J. P., F. C. MacKintosh,T. C. Lubensky \& Paul A. Janmey (2005). "Nonlinear elasticity in biological gels." Nature 435(112): 191-194.

3. Marek Cieplak, T. X. H., and Mark O. Robbins (2002). "Thermal Folding and Mechanical Unfolding Pathways of Protein Seconsary Structure." PROTEINS: Structure, Function, and Genetics 49: 104-113. 4. Pedro C Andia, F. C. a. G. L. G. (2006). "A classical mechanics approach to the determination of the stress-strain response of particle systems." MODELLING AND SIMULATION IN MATERIALS SCIENCE AND ENGINEERING 14: 741-757.

5. Buehler, M. J. (2006). "Mechanics of Protein Crystals: Atomistic Modeling of elasticity and Fracture." Journal of Computational and Theoretical Nanoscience 3: 670-683. 
6. J. Andrew McCammon, S. C. H. (1988). "Dynamics of proteins and nucleic acids." Cambridge University Press.

7. Weiner, J. H. (2002). "Statistical Mechanics of Elasticity (2nd Edition)." DOVER PUBLICATIONS, INC. Mineola, NewYork. 